Case Report

\title{
Surgical Treatment of Depressed Scar: A Simple Technique
}

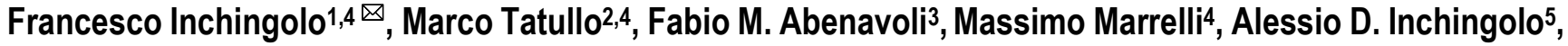 Roberto Corelli 6 , Angelo M. Inchingolo7, Gianna Dipalma ${ }^{4}$}

1. Department of Dental Sciences and Surgery, General Hospital, Bari, Italy

2. Department of Medical Biochemistry, Medical Biology and Physics, General Hospital, Bari, Italy

3. Department of "Head and Neck Diseases", Hospital "Fatebenefratelli", Rome, Italy

4. Department of Maxillofacial Surgery, Calabrodental, Crotone, Italy

5. Department of Dental Sciences and Surgery, General Hospital, Bari, Italy

6. Department of Maxillofacial Surgery, General Hospital, Bari, Italy

7. Department of Surgical, Reconstructive and Diagnostic Sciences, General Hospital, Milano, Italy

Corresponding author: Prof. Francesco INCHINGOLO, Piazza Giulio Cesare - Policlinico 70124 - Bari. E-mail: f.inchingolo@tin.it - f.inchingolo@doc.uniba.it. Tel.: 00390805593343 - Infoline: 00393312111104 - Fax: 00390883347794

() Ivyspring International Publisher. This is an open-access article distributed under the terms of the Creative Commons License (http://creativecommons.org/ licenses/by-nc-nd/3.0/). Reproduction is permitted for personal, noncommercial use, provided that the article is in whole, unmodified, and properly cited.

Received: 2011.03.29; Accepted: 2011.06.10; Published: 2011.06.18

\begin{abstract}
Scar formation is a process consequent to the healing of soft tissues after a trauma. However, abnormal or disturbed collagen production can cause anomalies of the cutaneous surface and textural irregularities. In the presence of a depressed scar in deep tissue, we began to use a new simple technique. In the presence of adherent scars, a small incision is performed so that an undermining scissor can enter inside. The entire cicatricial area is undermined on a subcutaneous plane which, by separating the deep scar from the superficial one, completely frees it from the present adhesions so that the existing depression is totally eliminated. In order to avoid the recreation of relapses, stitches formed in a U-shape are made in Nylon or Monocril 2-3/0 are made with a large needle and are placed close together so that a wide aversion is achieved at the margins of the scar and a deep wound closure is obtained by adhering to the undermined tissue. These stitches will then be removed about 2 weeks later.
\end{abstract}

Key words: Depressed scar, plastic surgery, subcision technique

\section{INTRODUCTION}

Scar formation is a process consequent to the healing of soft tissues after a trauma. However, abnormal or disturbed collagen production can cause anomalies of the cutaneous surface and textural irregularities. A cosmetically acceptable scar is often at the level with the surrounding skin, a good color match, soft, and narrow. Favorable lines of closure are usually within or parallel to relaxed skin tension lines: lines due to dynamic action of the underlying musculature. $^{1}$

The scar abnormality will guide the choice of treatment technique. The surgical strategy selected should be based on a correct evaluation of the scar's characteristics. In addition, while any scar with a suboptimal appearance can be revised, greatest patient satisfaction is achieved with realistic expectations. $^{2}$

\section{CASE REPORT}

In the presence of a depressed scar in deep tissue, we began to use a technique which I believe is interesting to present. In fact, there are cicatricial aftereffects that, though presenting a satisfactory aspect of the scar due to the presence of an adhesion of the 
skin to the underlying plane, disfigure somewhat in a disagreeable manner and therefore reconstructive plastic surgery should be performed (Fig.1). Thus, in these cases especially when there is not a lack of tissue secondary to trauma as for example the suffering of sub-cutaneous adipose tissue, it would be sufficient to eliminate the single adhesion without reopening the entire scar again.

Therefore, with this objective in mind, in the presence of adherent scars, after having infiltrated the whole area with a solution of Lidocaine $0.5 \%$ and Epinephrine 1:200,000 very close to one of the two edges of the scar, a small incision is performed so that an undermining scissor can enter inside (Fig.2).

The entire cicatricial area is undermined on a subcutaneous plane which, by separating the deep scar from the superficial one, completely frees it from the present adhesions so that the existing depression is totally eliminated (Fig.3).

At the end of this manoeuvre, in order to avoid the recreation of relapses, stitches formed in a U-shape are made in Nylon or Monocril 2-3/0 are made with a large needle and are placed close together so that a wide aversion is achieved at the margins of the scar and a deep wound closure is obtained by adhering to the undermined tissue (Fig.4). These stitches will then be removed about 2 weeks later. Afterwards, the patient should start to carry out massages on the entire treated area using moisturizing cream to aid the mobility of the recuperated tissue.

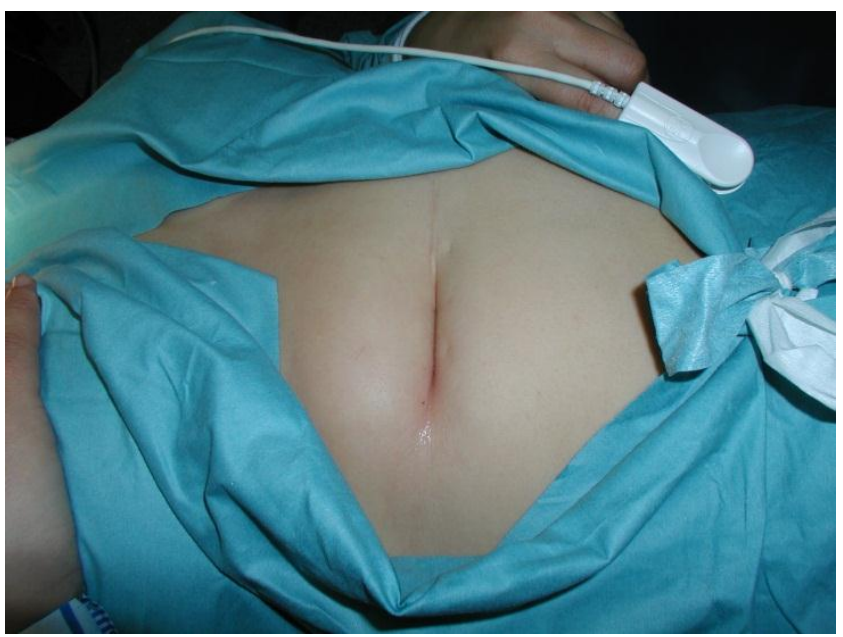

Fig.1: An example of a depressed scar of the abdomen.

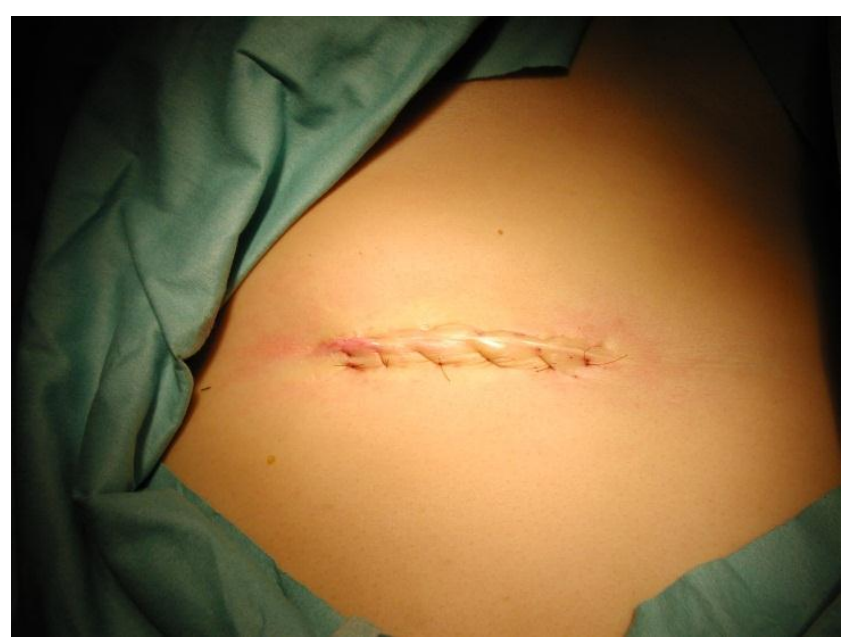

Fig.4: The final result 


\section{CONCLUSIONS}

Subcision incision is a very effective technique for correcting atrophic scars. Essentially, the physician inserts a needle and sweeps it back and forth repeatedly to free the skin from the underlying scar tissue.

Normally, the dissection plane of subcision is rather superficial: it is a subdermal dissection performed successfully in the treatment of acne scars. In this novel technique, we perform a deeper dissection plane and it is designed in order to remove adhesions that attach the skin to the floor below. Infact, the use of the stitches in depth is performed in order to prevent that the adhesion can recreate again: this relapse could promote the formation of a layer of reactive collagen in the region below the treated area.

We therefore believe that this technique can be utilized as a simple and safe technique that brings great improvement to the treatment of depressed scars.

\section{AUTHORS' CONTRIBUTIONS}

FI: participated in the surgical treatment and in the follow-up of this patient. MT: drafted the manuscript and reviewed the literature. FMA: participated in the surgical treatment and in the follow-up of this patient. MM: participated in the design of this case study and in the follow-up of this patient. ADI: revised the literature sources. RC: participated in the surgical treatment and in the follow-up of this patient. AMI: documented this case report with digital pictures. GD: participated in the follow-up of this patient. All the authors read and approved the final manuscript.

\section{CONSENT STATEMENT}

Written informed consent was obtained from the patient for publication of this case report and accompanying images.

\section{Conflict of Interest}

The authors have declared that no conflict of interest exists.

\section{References}

1. Thomas JR, Prendiville S. Update in scar revision. Facial Plast Surg Clin North Am 2002;10(1):103-11

2. Jacob CI, Dover JS, Kaminer MS. Acne scarring: a classification system and review of treatment options. J Am Acad Dermatol 2001;45(1):109-17 